\title{
A survey of flares and current helicity in active regions
}

\author{
S.D. Bao, H.Q. Zhang, G.X. Ai, and M. Zhang \\ Beijing Astronomical Observatory, Chinese Academy of Sciences, Beijing 100080, China \\ National Astronomical Observatories, Chinese Academy of Sciences, Beijing 100012, China
}

Received February 9; accepted June 9, 1999

\begin{abstract}
We examine the spatial and temporal relationship between chromospheric $\mathrm{H}_{\beta}$ flares and photospheric current helicities in active regions. All of the data were obtained by the vector magnetograph system at Huairou Solar Observing Station of Beijing Astronomical Observatory. We focus our analysis on NOAA Active Region 6233, which was observed on 30 August 1990. The result shows that rapid and substantial changes of distribution of current helicity in an area or in its vicinity are most likely to trigger flares, but no compelling correlation between peaks of current helicity and flare sites. Furthermore, we study the influence of flares on helicity for several other active regions. We find that the time variations of current helicity in the active regions with highly productive flares are more significant than those of the poorly flare-productive active regions, and that the magnitude of current helicity does not always decrease after flares. Therefore we conclude that the rate of variation of current helicity may be considered as an indicator of flare activity.

In this paper we describe the specification of the instrumentation and data used, and we describe the methods for computing helicity. The noises and errors in our data are estimated to be at the $2-3 \sigma$ level.
\end{abstract}

Key words: Sun: activity — Sun: flares — Sun: magnetic fields

\section{Introduction}

The source of energy of solar flares is generally believed to be due to an excess of energy in non-potential magnetic structures. Storage of this energy is perceived as a result of an increasing deformation of magnetic loops initially in a more potential configuration in the upper atmosphere. However, from the observational point of view, how to

Send offprint requests to: S.D. Bao

Correspondence to: bshd@sun10.bao.ac.cn extract the non-potential component and how to describe the non-potentiality more accurately, are still open questions.

While studying variations of magnetic structures connected with a flare, magnetic shear is of special interest. This concept was firstly recognized from the twisted appearance of $\mathrm{H}_{\alpha}$ fibrils or filaments observed during flare activity (Zirin \& Tanaka 1973). A quantitative evaluation of the shear in magnetic fields along the polarity inversion lines was achieved by defining the angular shear, $\triangle \phi$, as the azimuth difference at the photosphere between the potential magnetic fields, which fit the boundary conditions imposed by the observed line-of-sight fields, and the observed transverse fields (Hagyard et al. 1984). The buildup of magnetic shear should be expected to be associated with the overall development of a whole active region, such as different types of photospheric motions and new flux emergence. So it appears desirable to develop a method for describing the overall regional shear, not restricting the angular shear analysis to the magnetic inversion lines. A number of papers were devoted to exploring this research field (Gary et al. 1987; Wang 1992a; Ambastha et al. 1993; Fontenla et al. 1995; Zhang 1995; Wang et al. 1996), but examples of angular shear increase or decrease after major flares had equal shares.

Moreton \& Severny (1968) made pioneer observations of the relationship between $\mathrm{H}_{\alpha}$ emission in flares and active region electric currents. Thirty flares were studied, and it was found that about $80 \%$ of the initial brightenings in $\mathrm{H}_{\alpha}$ coincided with the principal sites of vertical currents, to within the $6^{\prime \prime}$ coalignment accuracy of their data sets. With greatly improved observations in quality, vector magnetograms in the photosphere were then used to further examine such a spatial relationship between flares and vertical currents (Lin \& Gaizauskas 1987; Canfield et al. 1993; de La Beaujardière et al. 1993; Leka et al. 1993; Metcalf et al. 1994; Wang et al. 1994; Zhang 1995, 1997.) Unfortunately, such an attempt failed to give any final conclusions. 
Gorbachev \& Somov (1988) were the first who studied a quantitative link between observed chromospheric flare kernels and the magnetic topology of the host active region. Since then, there has been a broad interest in the study of magnetic topology in terms of separatrices and separators (Mandrini et al. 1991, 1993, 1995; Démoulin et al. 1993, 1994; van Driel-Gesztelyi et al. 1994; Bagalá et al. 1995; Wang 1997; Wang et al. 1999). In all these studies, flare kernels were found at the intersection of the separatrices with the chromosphere, the kernels being magnetically connected to each other and to a common region around the separator.

We do know that helicity is a topological parameter which describes the complexity of an object and that it is relevant to the effects of twist, writhe and linkage. Magnetic helicity characterizes the topological complexity of a magnetic field. The main advances in magnetic helicity of the solar atmosphere were presented in recent review papers (Ricca \& Berger 1996; Low 1996; Canfield \& Pevtsov 1998). The most interesting result is the so-called hemispheric chirality rule — various magnetic patterns (e.g., photospheric net helicity in active regions, sunspot whirls, chromospheric filaments, sheared coronal loops, and interplanetary magnetic clouds) in the northern hemisphere show predominantly a left-handed twist while in the southern hemisphere they show a right-handed twist (see Seehafer 1990; Pevtsov et al. 1995; Zirker et al. 1997; and references therein). Bao \& Zhang (1998) computed the current helicities in 422 active regions, and found that about $80 \%$ of the regions in the northern (southern) hemisphere have negative (positive) helicity. This result confirms the above hemispheric sign asymmetry. The handedness shown in all these large-scale patterns seems to support the solar dynamo operating in the interface between the solar convection and radiation zones. More recently, Zhang \& Bao (1998, 1999) also studied the latitudinal variation of current helicity of active regions and the longitudinal distribution of the active regions with a reversedsign helicity during the solar cycle 22 . In the present paper, our primary purpose is to study magnetic activity before and during solar flares from the viewpoint of helicity.

In the next section, the instrumentation and analysis procedures for this study are described. In Sect. 3, we describe the definition of magnetic and current helicity, as well as the methods for computing them from vector magnetograms. In Sect. 4, the spatial and temporal relationship between flare occurrence and current helicity is examined in detail. Finally, the results are discussed in Sect. 5 .

\section{Observational techniques}

The database used in our study was obtained with the Solar Magnetic Field Telescope (SMFT), an imaging vector magnetograph (Ai \& Hu 1986) installed at the Huairou
Solar Observing Station (HSOS) of Beijing Astronomical Observatory. The SMFT consists of a $35 \mathrm{~cm}$ vacuum telescope, a $1 / 8 \AA$ Lyot birefringent filter with three sets of KD*P crystal modulators, a CCD camera with $512 \times 512$ pixels, and an image processing system controlled by a computer. It works in either of two spectral lines: Fe I $5324.19 \AA$ (photosphere) and $\mathrm{H}_{\beta} 4861.34 \AA$ (chromosphere). The field of view is about $5.23^{\prime} \times 3.63^{\prime}$. The temporal and spatial resolution of the HSOS vector magnetograms depends on the number of video frames that are added to produce them. Each magnetogram used in this paper is the sum of 256 individual frames for both lineof-sight and transverse fields, corresponding to a temporal resolution of about $5 \mathrm{~min}$ and a spatial resolution of 2 arcsec.

The inference of vector magnetic fields in the photosphere is on the basis of narrow-band images (filtergrams) of the four Stokes parameters $I, V, Q$ and $U . I$ is always the direct intensity, derived from either the sum of two circularly polarized images used for line-of-sight field measurements, or the sum of two linearly polarized images used for transverse field measurements. $V$ is the difference between the left and right circularly polarized images taken by shifting the bandpass at $-0.075 \AA$ from the line center. $Q$ is the difference between two orthogonal linearly polarized images in a certain azimuthal direction. $U$ is the same as $Q$, but with a $45^{\circ}$ change in the azimuthal direction. When $Q$ and $U$ are taken, the filter bandpass is switched to the line center for achieving maximum sensitivity. The line-of-sight component $B_{1}$ and transverse component $B_{\mathrm{t}}$ of magnetic fields are given by

$B_{\mathrm{l}}=C_{\mathrm{l}} \frac{V}{I}, \quad B_{\mathrm{t}}=C_{\mathrm{t}}\left(\frac{Q^{2}}{I^{2}}+\frac{U^{2}}{I^{2}}\right)^{\frac{1}{4}}$,

where $C_{\mathrm{l}}$ and $C_{\mathrm{t}}$ are calibration coefficients relating the line-of-sight and transverse components of magnetic fields to the circular and linear degree of polarization, respectively. Determining the coefficients is very complicated and difficult. Both theoretical and empirical methods are used to calibrate the HSOS vector magnetograms. Some details have been described by Wang et al. (1996). The azimuthal angle of the transverse field is

$\varphi=\frac{1}{2} \tan ^{-1}\left(\frac{U}{Q}\right)$

It should be mentioned that the magneto-optical effect (Faraday rotation) has been evaluated by theoretical calculations and found to be insignificant (Wang et al. 1996; and references therein). Several tests were made particularly to compare the measured field azimuth for a few sunspots when switching the bandpass from line center to line wings, and the differences in the observed azimuth were less than $10^{\circ}$. The contamination of the relatively weak linear polarization signal by the stronger circular polarization signal (cross-talk) for the HSOS vector magnetograms is not significant. From joint vector magnetograph observations at Big Bear, Huairou, and Mees Solar 
Observatories, Wang et al. (1992b) concluded that the cross-talk in the HSOS magnetograms is only about $2 \%$. In addition, the Fe $5324.19 \AA$ line used by the SMFT is a broad line with a width of approximately $0.334 \AA$, and its Landé factor is 1.5 . For such a strong and broad line, not only the Zeeman saturation and Doppler shift are not important, but also this line is not very sensitive to temperature. Numerical simulations show that when $B_{1}$ is $3000 \mathrm{G}$, the saturation effect can only cause a $3 \%$ relative error for the bandpass location of $-0.075 \AA$ to the line center (Ai et al. 1982). For the transverse field measurements, as long as the field strength is less than $2000 \mathrm{G}$, the linear relation in Eq. (1) will hold when the bandpass is less than $0.03 \AA$ from the line center. Although it is impossible to completely eliminate all these errors, some corrections have been made by adjusting the calibration coefficients of magnetic fields.

The $180^{\circ}$ azimuthal ambiguity in determining the transverse field direction is an intrinsic defect of Zeeman effect (Harvey 1969). The only way to resolve this problem at present is to introduce an additional constraint on the field azimuth either from independent observations, such as the history of the field evolution and the orientation of chromosphere fibrils, or from other theoretical considerations, such as the potential field approximation. For each magnetogram, we resolved this ambiguity following the method described by Wang et al. (1994).

To reduce the noise, a $3 \times 4$ smooth average of $V, Q$ and $U$ parameters was made. The noise level in all the lineof-sight magnetograms shown is $10 \mathrm{G}$. For the transverse field measurements, the blending of $\pi$ and $\sigma$ polarization components over the spectral bandpass used diminishes the linear polarization signal; this limits the sensitivity of $B_{\mathrm{t}}$ to $\sim 100 \mathrm{G}$.

All vector magnetogram data used in this paper were taken with favorable weather and seeing conditions during the time in which active regions were located near the central meridian. The projection effects of high-latitude active regions were removed according to the formulae given by Gary \& Hagyard (1990).

\section{Magnetic and current helicity}

\subsection{Helicity definition}

The density of magnetic helicity is defined as

$h_{\mathrm{m}}=\mathbf{A} \cdot \mathbf{B}$,

where $\mathbf{A}$ is a vector potential for magnetic field $\mathbf{B}$ (Moffatt 1978). In general, $h_{\mathrm{m}}$ determines the number of linkages of magnetic field lines. The total magnetic helicity in a domain $\mathcal{D}$ is

$H_{\mathrm{m}}=\iiint_{\mathcal{D}} h_{\mathrm{m}} \mathrm{d} V$

If space is divided into a collection of flux tubes, the helicity integral contains contributions from internal structure within a flux tube, such as twist and writhe, and from external relations between flux tubes, such as knotting and linking. Consider a thin tube $T$ with local $\mathbf{B}(r, \theta, z)$ defined by its axial component $\mathbf{B}_{\mathrm{a}}=\left(0,0, B_{z}(r)\right)$ and meridional component $\mathbf{B}_{\mathrm{m}}=\left(0, B_{\theta}(r), 0\right)$, Moffatt \& Ricca (1992) showed that the total helicity of the thin tube $H_{\mathrm{m}}=\iiint_{T} \mathbf{A}_{\mathrm{a}} \cdot \mathbf{B}_{\mathrm{a}} \mathrm{d} V+2 \iiint_{T} \mathbf{A}_{\mathrm{m}} \cdot \mathbf{B}_{\mathrm{m}} \mathrm{d} V$, where first integral represents writhe $\mathcal{W}$ and second represents twist $\mathcal{T}$. For two untwisted closed flux tubes linked, the total magnetic helicity is given by $H_{\mathrm{m}}= \pm 2 \Phi_{1} \Phi_{2}$, where $\Phi_{1}$ and $\Phi_{2}$ measure the magnetic flux of the tubes, and the sign of $H_{\mathrm{m}}$ depends on the sense of linkage (Berger \& Field 1984).

For a closed magnetic system $\left(B_{\mathrm{n}}=\left.\mathbf{B} \cdot \hat{\mathbf{n}}\right|_{\sigma}=0\right)$, the variation of magnetic helicity with time is readily derived from the Maxwell equations, and can be written in the final form

$\frac{\mathrm{d} H_{\mathrm{m}}}{\mathrm{d} t}=-2 \iiint_{\mathcal{D}} \eta \mathbf{J} \cdot \mathbf{B} \mathrm{d} V$

here $\eta$ is the resistivity and $\mathbf{J}$ is the electric current density. Clearly, the total magnetic helicity is conserved in ideal $\mathrm{MHD}(\eta=0)$. Equation (5) may be rewritten as

$\frac{\mathrm{d} H_{\mathrm{m}}}{\mathrm{d} t}=-\frac{2 \eta}{\mu_{0}} H_{\mathrm{c}}$

$H_{\mathrm{c}}=\iiint_{\mathcal{D}} h_{\mathrm{c}} \mathrm{d} V, \quad h_{\mathrm{c}}=\mu_{0} \mathbf{J} \cdot \mathbf{B}=\mathbf{B} \cdot(\nabla \times \mathbf{B})$,

where $\mu_{0}$ is the vacuum permeability, $h_{\mathrm{c}}$ is the current helicity density, and $H_{\mathrm{c}}$ is the total current helicity. With analogy to the magnetic helicity, $H_{\mathrm{c}}$ describes the linkages of electric currents.

\subsection{Measure of current helicity}

The direct computation of magnetic helicity $H_{\mathrm{m}}$ is hindered by the fact that the magnetic vector potential $\mathbf{A}$ is unmeasured. However, under the assumption of a linear force-free field

$\nabla \times \mathbf{B}=\alpha \mathbf{B}$,

the magnetic helicity density will become

$h_{\mathrm{m}}=\alpha^{-1} B^{2}$.

Here $\alpha$ is the force-free field parameter, which can be observationally estimated (Seehafer 1990; Pevtsov et al. 1994, 1995). These authors suggest that the parameter $\alpha$ is a plausible helicity proxy to characterize the twist in active region magnetic fields. Similarly, the current helicity density may become also

$h_{\mathrm{c}}=\alpha B^{2}$.

There is, indeed, no reason to believe that the magnetic fields at photospheric levels are force-free. In this case we are unable to compute the magnetic helicity, but we can in principle determine the current helicity density according to Eq. (7). Unfortunately, our vector magnetograms observed only at a single height allow us to compute only the 
vertical component of current helicity, i.e., $B_{z} \cdot(\nabla \times \mathbf{B})_{z}$. This quantity may be expressed by

$h_{\mathrm{c}}=\mu_{0} B_{z} J_{z}$,

and

$J_{z}=\frac{1}{\mu_{0}}\left(\frac{\partial B_{y}}{\partial x}-\frac{\partial B_{x}}{\partial y}\right)$,

where $B_{z}, B_{x}$ and $B_{y}$ are the vertical and horizontal components of magnetic fields in heliographic coordinates, which are derived from both the observed line-of-sight field $B_{\mathrm{l}}$ and transverse field $B_{\mathrm{t}}$ (Venkatakrishnan et al. 1988).

In this paper we choose the formula (11) to compute current helicity, considering that, since only the vertical component of electric currents enters the solar upper atmosphere and determines its helicity, $\mu_{0} B_{z} J_{z}$ should play a leading role in current helicity (Bao \& Zhang 1998). We estimate errors by computing the standard deviation $\sigma_{J_{z}}$ of the current values in the set of pixels whose transverse fields are within the estimated uncertainty in $B_{\mathrm{t}}$; an electric current is considered to be significant only if its magnitude exceeds $3 \sigma_{J_{z}}$. Note that our analysis, in which we determine a single average value $<h_{\mathrm{c}}>$ for a whole active region, greatly decreases the influence of noise compared to that of a single-pixel measurement of $h_{\mathrm{c}}$. On the other hand, our choice to use the average value $\left\langle h_{\mathrm{c}}>\right.$ has a physical justification because there is often a pronounced overall twist in the active region.

\section{Relationship of flare occurrence to current helicity}

\subsection{Current helicity and flare kernels in AR 6233}

Active region 6233 was a flare-productive region which was located near the disk center (N13, W04) on August 30, 1990. Observations of this region, including a study of the morphological relationship between vertical electric currents and chromospheric flare phenomena, can be found in Wang et al. (1996) (also see de La Beaujardière et al. 1993); while the correlation of its magnetic separatrices with flare manifestations has been studied by Mandrini et al. (1995).

In this section we discuss how the distribution of current helicity in AR 6233 changes in the areas where flares occurred. To illustrate its general configuration, a photospheric image of the active region is shown in Fig. 1a; while Figs. $1 \mathrm{~b}$ and $1 \mathrm{c}$ are $\mathrm{H}_{\beta}$ filtergrams of two flares to be discussed, a SN/M1.2 flare at 01:58 UT and a SN/M1.0 flare at 04:34 UT on August 30. Figure 2 shows a time sequence of the distribution of current helicity from 00:57 UT to $06: 28$ UT on the day. Note that only significant current helicity densities $\left(\geq 10 \sigma_{h_{\mathrm{c}}}\right)$ are displayed in Fig. 2 . Thus, any little changes shown are thought to be due to a true change of current helicity itself rather than from noise or error. The bold contours in Fig. 2b and Fig. 2d represent the brightenings of the flare $F_{1}$ and the flare $F_{2}$, respectively. The $\mathrm{H}_{\beta}$ filtergrams of these two flares can be seen in Figs. 1b and 1c. For a convenient description, the active region is partitioned into three parts, panels $\mathrm{A}, \mathrm{B}$ and $\mathrm{C}$ (see Fig. 2a).

From Figs. 2a-2e we see that the distribution of current helicity in panel A hardly varies with time. Panel $A$ is a sunspot region where very strong magnetic fields are observed. Correspondingly, the values of current helicity in panel A are the maximum, but no flare kernel was observed in the panel. This indicates that sites of high current helicity density in active regions do not coincide with flare kernels.

For panel B, the distributions of current helicity in all figures except Fig. 2b are almost the same, as shown in panel A. The only difference among them is that the distribution of positive current helicity (solid contours) in panel B disappears in Fig. 2b. We infer that this phenomenon may be related to the flare $\left(F_{1}\right)$, which occurred near panel B at 01:58 UT. Such a relationship between significant changes of current helicity and flare occurrence is also found in panel C. From Figs. 2a-2d, we can see that the distribution of current helicity in panel $\mathrm{C}$ has obvious changes. During the course of the changes there are two flares occurring in panel $\mathrm{C}$. One of them, the flare $F_{2}$, is shown in Fig. 2d, and it occurred at 04:34 UT. On the other hand, we find that the distribution of current helicity in panel $\mathrm{C}$ has almost no changes between Figs. $2 \mathrm{~d}$ and 2e, while from 05:04 UT to 06:28 UT no flare was observed in panel C. In other words, this panel is quiet in the interval. Therefore, we conclude that rapid and substantial changes of current helicity distribution in an area or in its vicinity seem to be associated with flare eruptions, but in no-flaring regions, such changes are insignificant.

\subsection{Changes of current helicity in productive and poor flare regions}

In order to have sufficient evidence for the above conclusion, we further analyze some active regions whose characteristics are enumerated in Table 1 . These active regions are divided into two groups. One group is flare-productive, and the other shows little flare activity. Note that, compared to typical active regions, the selected flare-poor regions are relatively complicated.

Figure 3 shows the time series changes of current helicity imbalance, $\rho_{\mathrm{h}}$, in four flare-productive active regions. The quantity $\rho_{\mathrm{h}}$ is defined by

$\rho_{\mathrm{h}}=\frac{\sum h_{\mathrm{c}}(i, j)}{\sum\left|h_{\mathrm{c}}(i, j)\right|} 100 \%$,

where $h_{\mathrm{c}}(i, j)$ is the value of current helicity at a given pixel $(i, j)$, and the denominator represents the sum of the absolute values of all $h_{\mathrm{c}}(i, j)$ in the active region. $\rho_{\mathrm{h}}$ is actually a measure of imbalance of current helicity sign over a whole active region (Abramenko et al. 1996; 

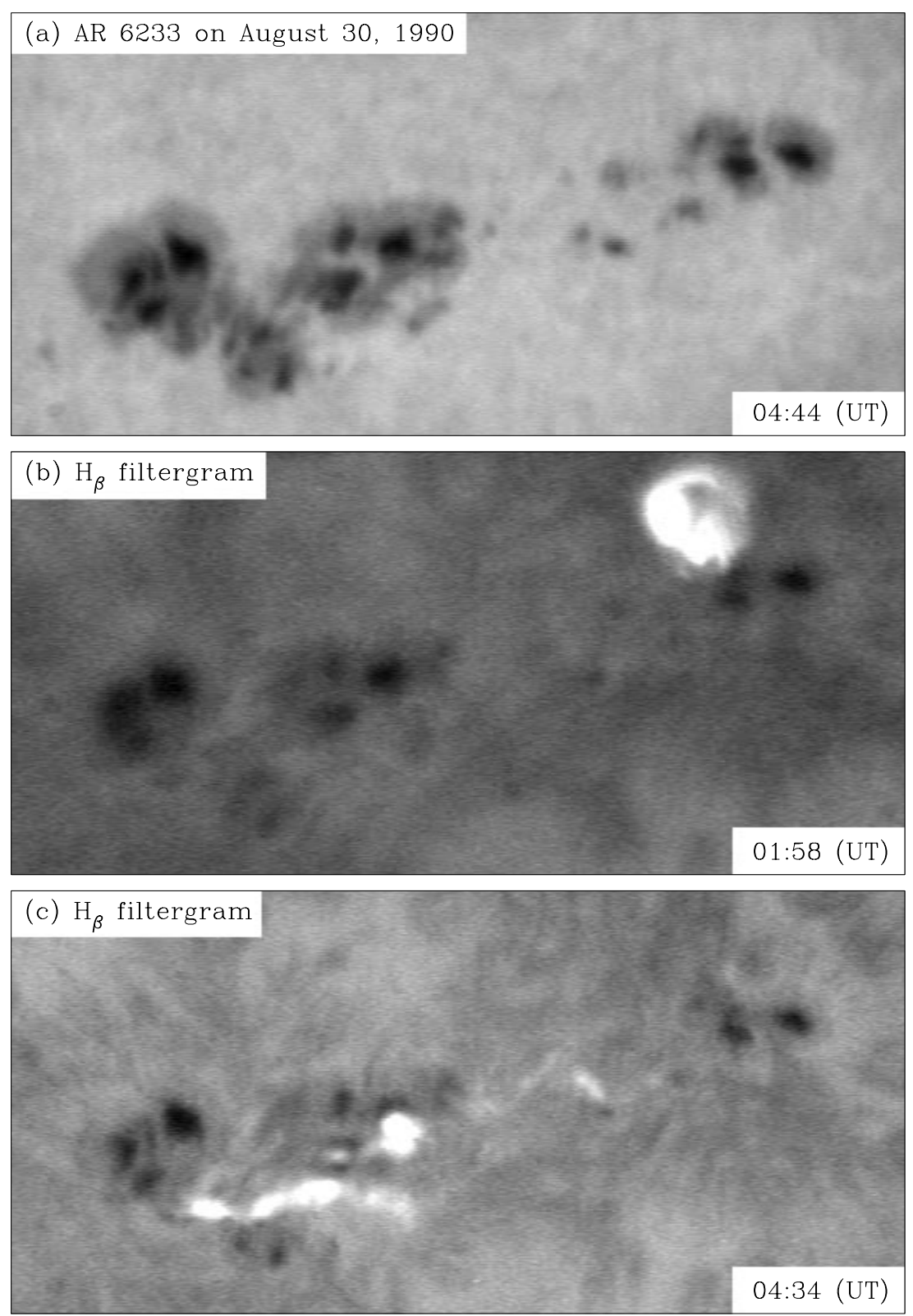

Fig. 1. a) A photospheric image of NOAA Active Region 6233 on 30 August 1990. b, c) $\mathrm{H}_{\beta}$ filtergrams of this region at 01:58 UT and 04:34 UT on the same day. The dark patches are sunspots, while the bright patches are flare kernels. North is at the top, and West is to the right

Table 1. Characteristics of selected active regions

\begin{tabular}{cclllc}
\hline \multirow{7}{*}{ NOAA/AR } & Date & Coordinate & Mag-class & Spot-class & Area $\left(10^{-6}\right.$ Hemi $)$ \\
\hline \multicolumn{7}{c}{ Flare-productive regions } \\
6233 & 30 Aug., 1990 & N13 W05 & BGD & FKC & 700 \\
6891 & 27 Oct., 1991 & S12 E18 & BGD & FKC & 2100 \\
7321 & 25 Oct., 1992 & S24 E06 & BGD & EKC & 1200 \\
7773 & 05 Sep., 1994 & S08 E05 & B & EKO & 470 \\
\hline 5612 & 03 Aug., 1989 & S17 W01 & B & DKI & 560 \\
5738 & 13 Oct., 1989 & N11 E05 & B & DKO & 460 \\
7496 & 09 May, 1993 & N14 E06 & BG & DAI & 300 \\
7903 & 30 Aug., 1995 & N03 E10 & B & CSO & 120 \\
\hline
\end{tabular}




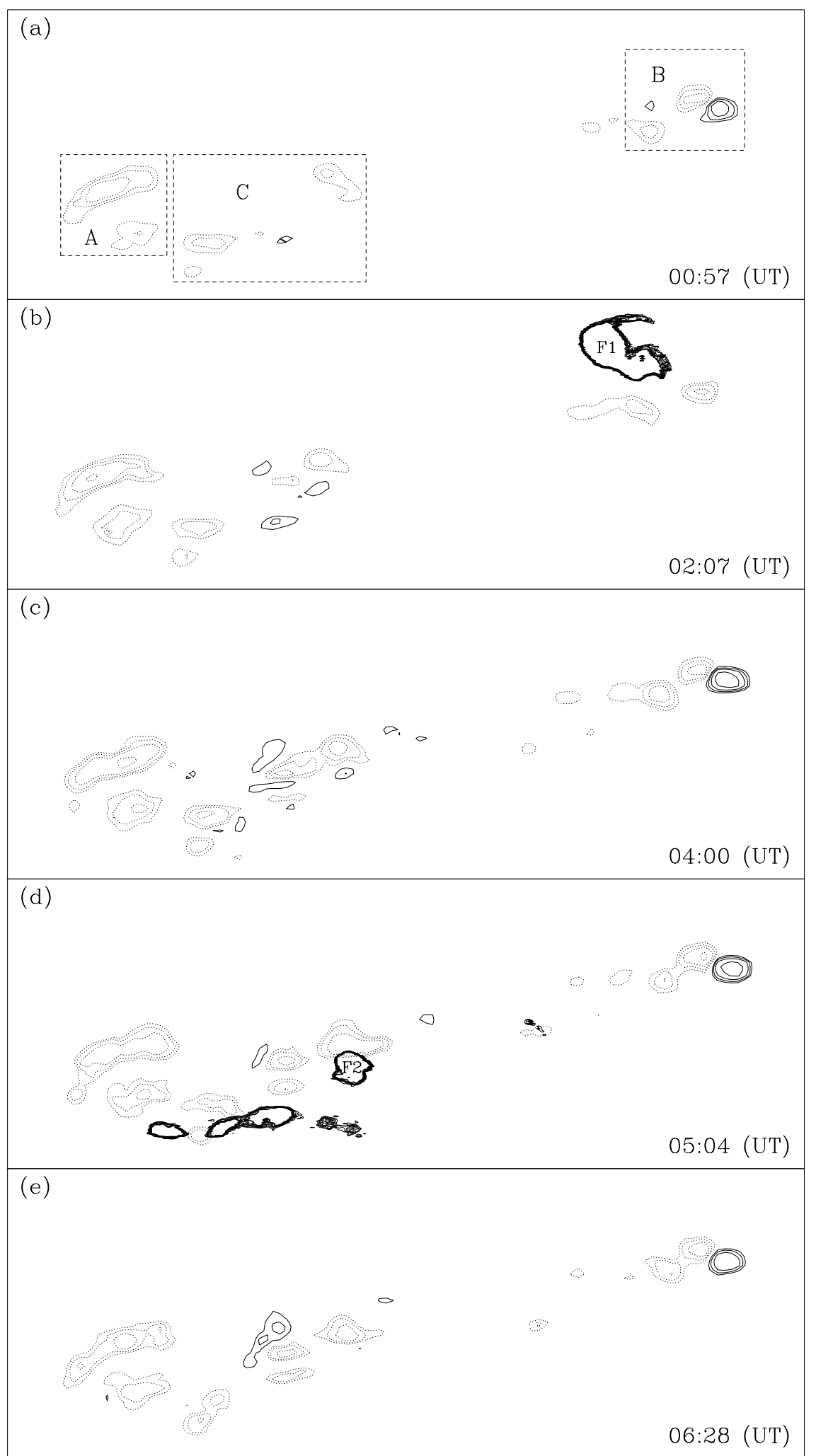

Fig. 2. Time sequence of the distribution of current helicity density in NOAA Active Region 6233 on 30 August 1990. The solid (dotted) contours correspond to positive (negative) current helicity of $0.07,0.12,0.20$, and $0.40 \mathrm{G}^{2} \mathrm{~m}^{-1}$. The bold contours represent two flares occurred at 01:58 UT and 04:34 UT respectively 

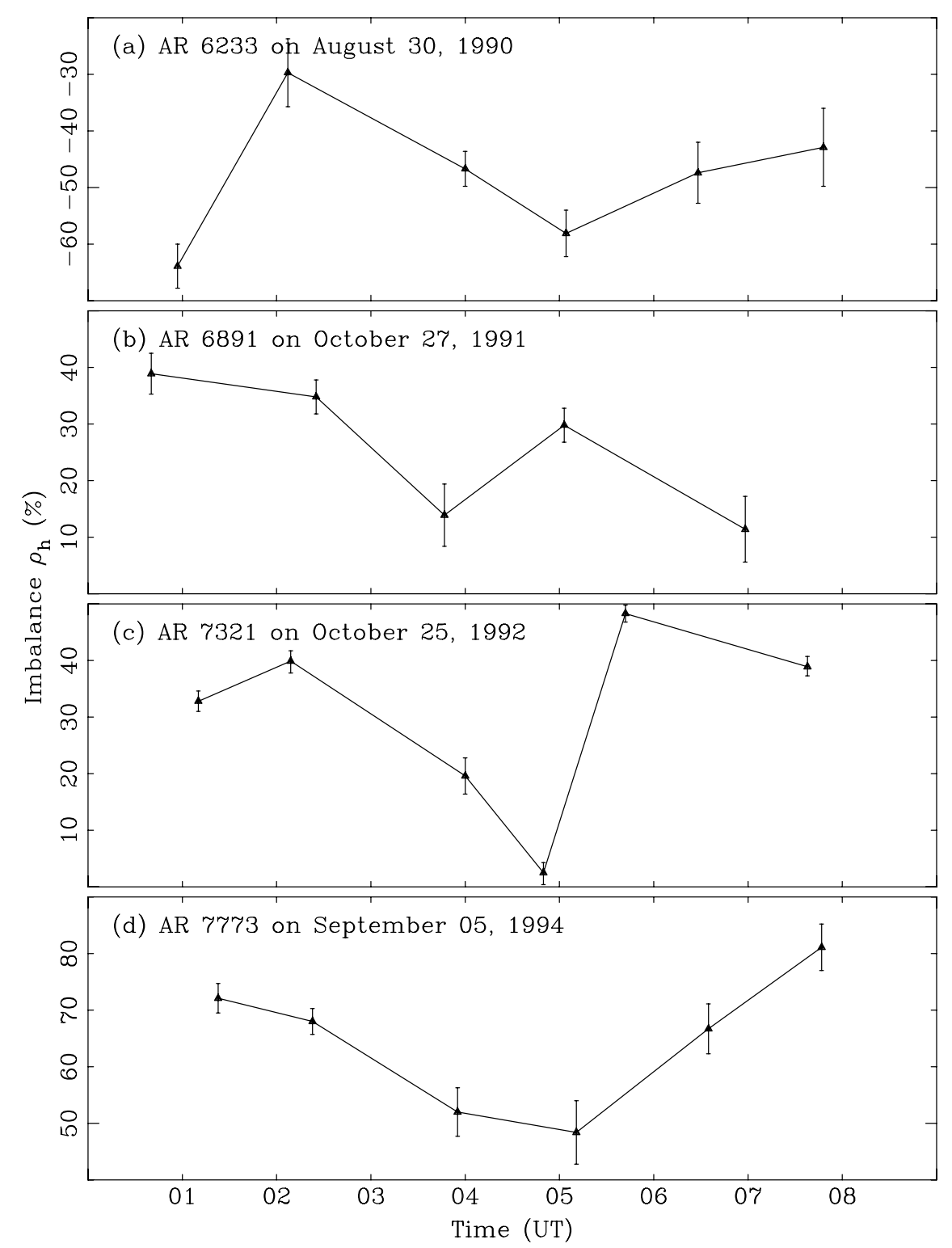

Fig. 3. Maps of the evolution of current helicity imbalance $\rho_{\mathrm{h}}$ in four flare-productive active regions (NOAA 6233, NOAA 6891, NOAA 7321 and NOAA 7773). Solid triangles indicate the times of magnetograms recorded. Obvious variations of current helicity for a whole active region are shown

Bao \& Zhang 1998), and its significant changes undoubtedly reflect that the distribution of current helicity density in an active region does vary with time. Along with the obvious variations of $\rho_{\mathrm{h}}$ in Figs. 3a-3d, some flares occurred in succession (see the arrows in Fig. 5). Similarly, Fig. 4 shows the time series changes of $\rho_{\mathrm{h}}$ in poorly flareproductive active regions. By comparing these two figures, we find that the variations of $\rho_{\mathrm{h}}$ are much more significant in Fig. 3 than those in Fig. 4. From this, we may infer that for any active regions, even complicated ones, if the temporal variations of current helicity are insignificant, the frequency of flaring activity is very low; on the contrary, even for ordinary active regions, if their $\rho_{\mathrm{h}}$ is changeable, correspondance to the frequency is high.
Note that the average current helicity $<h_{\mathrm{c}}>$ for a whole active region changes as obviously as the current helicity imbalance $\rho_{\mathrm{h}}$ in flare-productive active regions, as shown in Fig. 5. From this figure, we can clearly see that the magnitude of current helicity does not always come down after a flare. Flaring activity seems to be globally associated with the rate of variations in $\left\langle h_{\mathrm{c}}\right\rangle$. However, there is not a one to one relation between flare activity and variation of $\left\langle h_{\mathrm{c}}\right\rangle$. This result does not agree with that of Pevtsov et al. (1995). We argue that the rate of variation of current helicity in active regions is more closely related to solar flares, and it may better characterize the non-potentiality of active regions rather than the values of current helicity. 

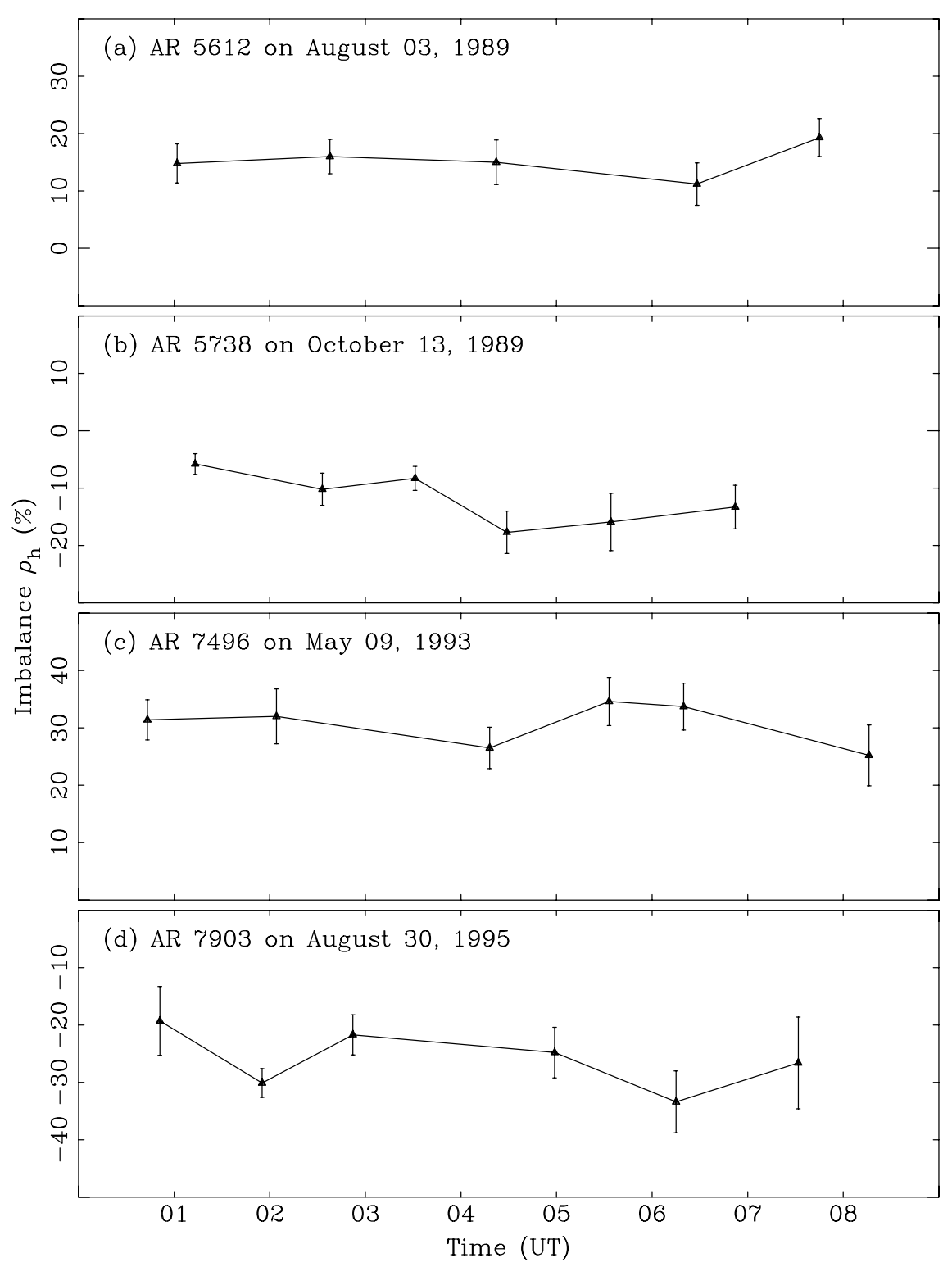

Fig. 4. Maps of the evolution of current helicity imbalance $\rho_{\mathrm{h}}$ in four flare-poor active regions (NOAA 5612, NOAA 5738, NOAA 7496 and NOAA 7903). Solid triangles indicate the times of magnetograms recorded. In these regions, variations of $\rho_{\mathrm{h}}$ are insignificant

\section{Conclusions and discussion}

The above observations and analysis lead us to believe that, (1) rapid and substantial changes of current helicity distribution in an area or in its vicinity probably lead to flare eruptions; (2) active regions in which average current helicity has a significant change show more flare activity than typical active regions; (3) no clear correlation between the peak values of current helicity and flare kernels; (4) the rate of variation of current helicity may better characterize the non-potentiality of active region magnetic fields - perhaps it can provide us with more information than other parameters, such as angular shear or vertical current.
We know that the magnetic helicity in the solar atmosphere comes mainly from the deeper layers of the Sun, and is accumulated during the course of magnetic activity. The accumulation will increase the magnetic complexity and magnetic free energy in the lower corona, and lead to flare eruptions. Taylor (1974) predicted that as a magnetized plasma relaxes, its magnetic field will evolve toward a force-free state, conserving total magnetic helicity. If Taylor's postulate is applicable for the solar atmosphere, the magnetic energy stored in the solar atmosphere evolves toward small spatial scales and dissipates much faster than magnetic helicity, which cascades toward larger spatial scales. However, this conservation of magnetic helicity is only in a global sense, it may be redistributed locally 


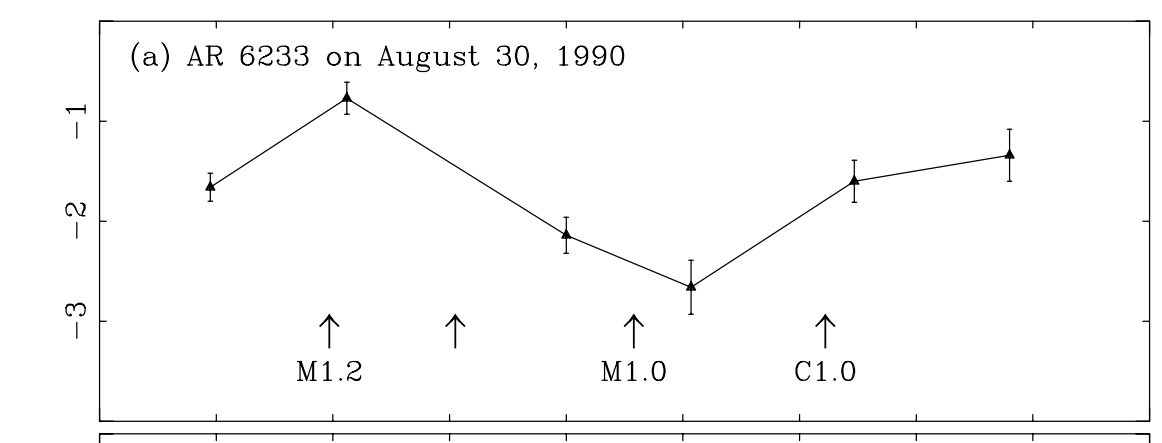

(b) AR 6891 on October 27, 1991

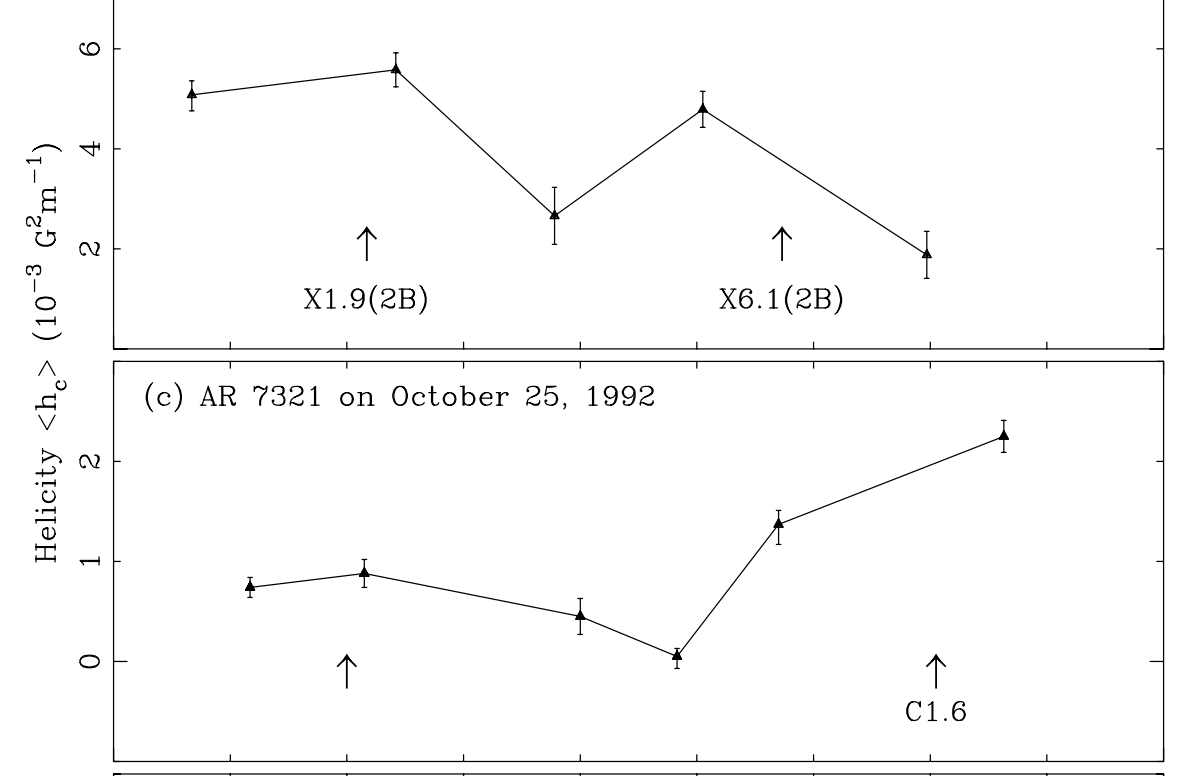

(d) AR 7773 on September 05, 1994

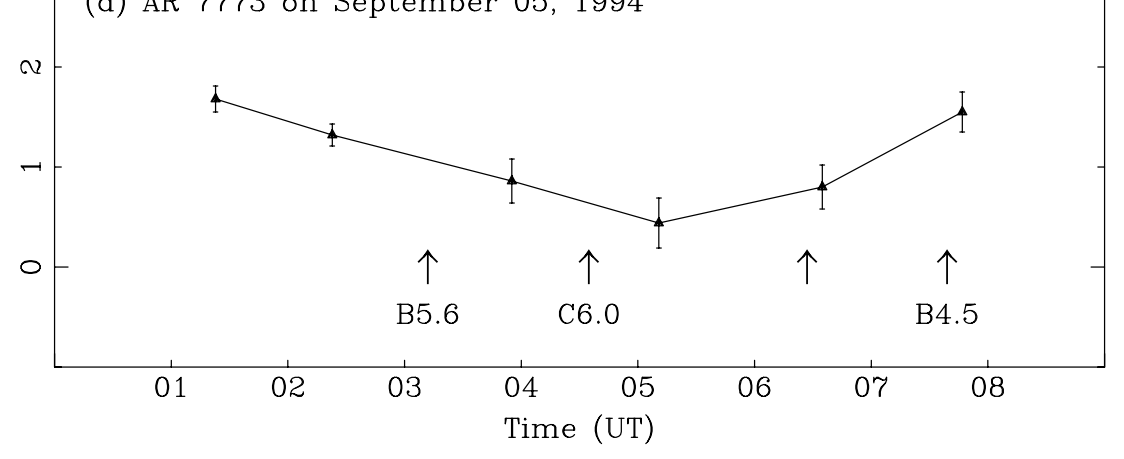

Fig. 5. Variations of the average current helicity $\left\langle h_{\mathrm{c}}>\right.$ of the photospheric magnetic fields as a function of time in four flare-productive active regions (NOAA 6233, NOAA 6891, NOAA 7321 and NOAA 7773). Arrows indicate start times of flares

between magnetic systems as a result of reconnection. Such a helicity exchange may lead to instability in a system with higher helicity. Therefore, solar flares may be understood in the framework of current helicity change processes. Of course, photospheric shear motions besides reconnection may also cause gradual buildup of twist in active regions. Which of these two processes prevails on the Sun? This is beyond the scope of this paper.

In fact, many solar observations (e.g., the soft X-ray images and chromospheric filaments) imply that twist is found in magnetic structures that show a tendency to erupt. In the present paper, we try to discuss how the non-potential structures of photospheric magnetic fields in active regions change before and after flares, from the viewpoint of helicity. The results obtained here are preliminary since we do not have 3 -dimensional observation data and study changes of current helicity distribution only in the photosphere. Although the helicity approach has not yet been widely applied in the study of flares, we think that the study of the influence of flares on 
helicity is very important for understanding the magnetic field structure and dynamic processes in the solar atmosphere. One should further research this topic, using a much larger sample.

Acknowledgements. We are indebted to Dr. Tongjiang Wang for helpful software and discussion. We also thank the referee for suggestions for improving the paper. This work was supported by the National Nature Science Foundation of China under grant 19791090.

\section{References}

Abramenko V.I., Wang T.J., Yurchishin V.B., 1996, Sol. Phys. 168,75

Ai G.X., Hu Y.F., 1986, Acta Astron. Sin. 27, 173

Ai G.X., Li W., Zhang H.Q., 1982, Acta Astron. Sin. 23, 39

Ambastha A., Hagyard M.J., West E.A., 1993, Sol. Phys. 148, 277

Bagalá L.G., Mandrini C.H., Rovira M.G., et al., 1995, Sol. Phys. 161, 103

Bao S.D., Zhang H.Q., 1998, ApJ 496, L43

Berger M.A., Field G.B., 1984, J. Fluid Mech. 147, 133

Canfield R.C., de La Beaujardière J.-F., Fan Y.H., et al., 1993, ApJ 411, 362

Canfield R.C., Pevtsov A.A., 1998, in "Synoptic Solar Physics", Balasubramaniam K.S., Harvey J.W., Rabin D.M. (eds.), ASP Conf. Ser. 140, 131

de La Beaujardière J.-F., Canfield R.C., Leka K.D., 1993, ApJ 411,378

Démoulin P., Mandrini C.H., Rovira, M.G., et al., 1994, Sol. Phys. 150, 221

Démoulin P., Van Driel-Gesztelyi L., Schmieder B., et al., 1993, A\&A 271, 292

Fontenla J.M., Ambastha A., Kalman B., Csepura G., 1995, ApJ 440, 894

Gary G.A., Hagyard M.J., 1990, Sol. Phys. 126, 21

Gary G.A., Moore R.L., Hagyard M.J., Haisch B.M., 1987, ApJ 314,782

Gorbachev V.S., Somov B.V., 1988, Sol. Phys. 117, 77

Hagyard M.J., Smith J.B., Jr. Teuber D., West E.A., 1984, Sol. Phys. 91, 115

Harvey J.W., 1969, NCAR Cooperative Thesis No. 17,
University of Colorado

Leka K.D., Canfield R.C., McClymont A.N., et al., 1993, ApJ 411,370

Lin Y.Z., Gaizauskas V., 1987, Sol. Phys. 109, 81

Low B.C., 1996, Sol. Phys. 167, 217

Mandrini C.H., Démoulin P., Hénoux J.C., et al., 1991, A\&A 250,541

Mandrini C.H., Démoulin P., Rovira M.G., et al., 1995, A\&A 303, 927

Mandrini C.H., Rovira M.G., Démoulin P., et al., 1993, A\&A 272,609

Metcalf T.R., Canfield R.C., Hudson H.S., et al., 1994, ApJ 428,860

Moffatt H.K., 1978, Magnetic Field Generation in Electrically Conducting Fluids. Cambridge University Press, p.19

Moffatt H.K., Ricca R.L., 1992, Proc. R. Soc. Lond. A 439, 411

Moreton G.E., Severny A.B., 1968, Sol. Phys. 3, 282

Pevtsov A.A., Canfield R.C., Metcalf T.R., 1994, ApJ 425, L117

Pevtsov A.A., Canfield R.C., Metcalf T.R., 1995, ApJ 440, L109

Ricca R.L., Berger M., 1996, Phys. Today 12, 28

Seehafer N., 1990, Sol. Phys. 125, 219

Taylor J.B., 1974, Phys. Rev. Lett. 33, 1139

van Driel-Gesztelyi L., Hofmann A., Démoulin P., et al., 1994, Sol. Phys. 149, 309

Venkatakrishnan P., Hagyard M.J., Hathaway D.H., 1988, Sol. Phys. 115, 125

Wang H.M., 1992, Sol. Phys. 140, 85

Wang H.M., Varsik J., Zirin H., et al., 1992, Sol. Phys. 142, 11

Wang H.N., 1997, Sol. Phys. 174, 265

Wang J.X., Shi Z.X., Wang H.N., Lü Y.P., 1996, ApJ 456, 861

Wang T.J., Wang H.N., Qiu J., 1999, A\&A 342, 854

Wang T.J., Xu A.A., Zhang H.Q., 1994, Sol. Phys. 155, 99

Zhang H.Q., 1995, A\&A 304, 541

Zhang H.Q., 1997, A\&A 324, 713

Zhang H.Q., Bao S.D., 1998, A\&A 339, 880

Zhang H.Q., Bao S.D., 1999, ApJ 519, 876

Zirin H., Tanaka K., 1973, Sol. Phys. 32, 173

Zirker J.B., Martin S.F., Harvey K, Gaizauskas V., 1997, Sol. Phys. 175, 27 